\title{
UV-curing Behaviors of Dendritic Polyacrylates
}

\author{
Ken'ichi Aoki $^{a}$, Akiko Hiraoka ${ }^{b}$ and Kunihiro Ichimura ${ }^{a}$ \\ ${ }^{a}$ Center of Advanced photopolymers, Faculty of Science, Toho University, \\ 2-2-1 Miyama, Funabashi, Chiba 274-8510, Japan \\ ${ }^{b}$ Yokkaichi Laboratory, Hakuto Co., Ltd., Mie, 510-0007, Japan \\ k-aoki@sci.toho-u.ac.jp
}

\begin{abstract}
Keywords: Dendrimer, Polyacrylate, UV-curing, Photopolymerization, Polymerization shrinkage
\end{abstract}

\section{Introduction}

UV-curing systems have been extensively employed in various application fields as exemplified by coating, inks, adhesion and so forth. Multifunctional acrylate monomers giving rise to radical photopolymerization are generally used as UV-curable materials. Hyperbranched (HB) polyacrylates have been attracting recent interest because of improved UV-curing rates and low viscosity, as demonstrated by Hunt et al.[1]. Effects of chemical as well as physical properties of HB poly(meth)acrylates on UV-curing behavior were also reported [2,3]. On the other hand, it has been elucidated that commercially available HB polyols referred to<smiles>C=CC(=O)OCC(C)OCC(C)OCC(C)OCC(C)OC(=O)C=C</smiles>

TPGDA<smiles>CCOC(=O)C=CC(=O)OCCNC(=O)OCCSCC(=O)OC(C)CC</smiles>

Ac8

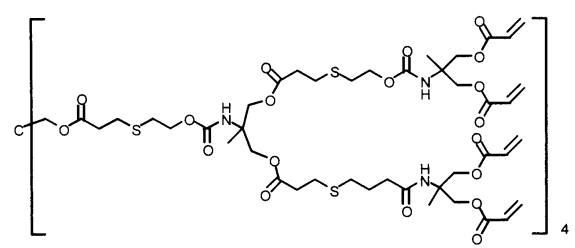

Ac16

Scheme 1. Chemical structures of TPGDA and dendritic $\operatorname{Ac}(\boldsymbol{n})(\boldsymbol{n}=\mathbf{8}, \mathbf{1 6})$ used in this study.
Boltorn $\mathrm{H} x$ exhibit wide polydispersity and the irregularity of $\mathrm{OH}$ groups consisting of terminal, dendritic and linear units [4]. Accordingly, HB polyacrylates studied so far may possess also wide polydispersity and irregular molecular structures. [3] Distinct difference of dendrimers from HB polymers exists in their well-defined molecular structures and narrow polydispersity so that improved properties including UV-curing rates and the reduced polymerization shrinkage are anticipated when dendritic polyacrylates are available.

We have recently developed the novel synthetic method of dendrimers called "alternate multi-addition (AMA)" process to afford dendritic polyacrylates with well-defined molecular structures in a large scale [5]. We report here some UV-curing behaviors of dendritic polyacrylates.

\section{Method}

Dendritic polyacrylates of $\operatorname{Ac}(n)(n=8$ and 16 , Scheme 1) [5] and conventional bifunctional monomer of tripropyleneglycol diacrylate (TPGDA, Scheme 1) were dissolved in THF with a photoinitiator (Ir-369), and the solutions were spincast on PET or $\mathrm{KBr}$ substrates. Resultant films were heated at $75{ }^{\circ} \mathrm{C}$ for $1 \mathrm{~min}$ to remove the solvent and exposed to $365 \mathrm{~nm}$ light (22 $\mathrm{mW} \mathrm{cm}$ ) to be subjected to FTIR measurements. For the determination of photosensitivity, a sample film was sandwiched between a couple of PET substrates to avoid the inhibitory effect of oxygen on photopolymerization. 


\section{Results and Discussion}

Dendritic polacrylates of Ac8 and Ac16 were synthesized according to our procedure [5]. The dendrimers and TPGDA were dissolved in THF, respectively, in the presence of $2.5 \mathrm{w} / \mathrm{wt} \%$ of Ir-369. Spin-casting of the dendrimer solutions on PET or $\mathrm{KBr}$ substrates, followed by drying, gave viscous films of 3-5 $\mu \mathrm{m}$ in thickness. UV irradiation of $365 \mathrm{~nm}$ light $\left(3-4 \mathrm{~J} \mathrm{~cm}^{-2}\right)$ brought about the hardening of the films under aerobic conditions. FTIR spectra revealed that the intensity of $v_{\mathrm{C}=\mathrm{C}}$ at around $1635 \mathrm{~cm}^{-1}$ decreased markedly upon UV irradiation. Amounts of acrylate moieties consumed by UV irradiation were estimated to be $c a$. $60-70 \%$ for both of Ac8 and Ac16.

In order to obtain further information on the UV-curing behavior, changes in pencil hardness of films were examined as a function of UV exposure doses under anaerobic conditions by placing a sample film between two PET films, and the results are depicted in Figure 1. It was found that the exposure energy to reach $2 \mathrm{H}$ in pencil hardness was estimated to be 700,83 and $75 \mathrm{mJcm}^{-2}$ for the films of TPGDA, Ac8 and Ac16, respectively. The photosensitivity of the films of dendritic polyacrylates is about ten-fold as high as that of the bifunctional monomer due to the local concentration of the acrylate moieties at the molecular surfaces, leading to the efficient activities on the radical polymerization. The polyacrylate dendrimers are also useful as

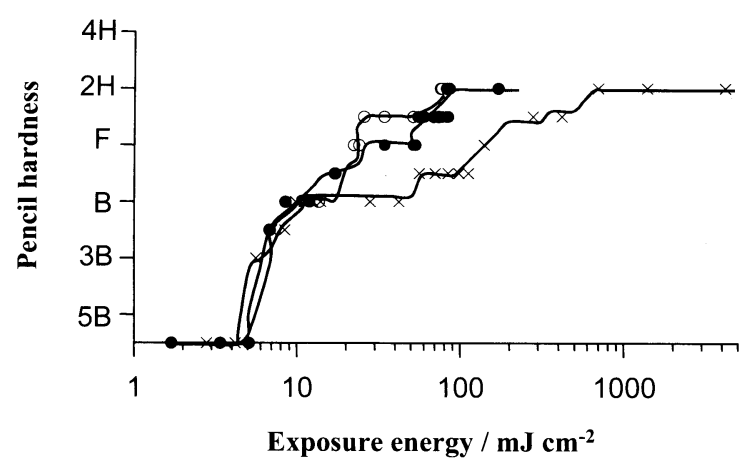

Figure 1. Pencil hardness of thin films of TPGDA (x), Ac8 (•) and Ac16 ( $\bigcirc$ ) as a function of exposure doses of $365 \mathrm{~nm}$ light. curing enhancers. For example, a 1:10 w/w mixture of Ac8 and 2-hydroxyethyl acrylate (HEA) was hardened by UV irradiation of a 427

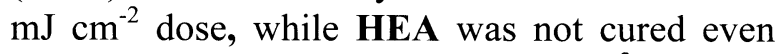
after prolonged UV irradiation $\left(6 \mathrm{~J} \mathrm{~cm}^{-2}\right)$.

Another interesting character on the dendritic photopolymers is remarkably low polymerization shrinkage. Polymerization shrinkage was estimated by measuring specific gravity before and after UV-irradiation. The degrees of shrinkage were estimated to be $3.0 \%$, $2.8 \%$ for Ac8 and Ac16, respectively, while $13.2 \%$ for conventional trimethylolpropane triacrylate. Note that $6.1 \%$ of shrinkage was observed for a hyperbranched polyacrylate (V\#1000, Osaka Organic Chemical Industry Ltd.). The reduction of shrinkage after UV-curing of dendritic polyacrylates is ascribable to the attachment of acrylate groups specifically at the termini of chains, which are folded due to the spherical molecular structure.

In summary, UV-curing performances of polyacrylate photopolymers were improved by using mono-dispersed dendritic backbones. High photoreactivity and low polymerization shrinkage can be simultaneously achieved, which contributes to the practical application of the UV-curable materials such as coating, adhesion and patterning.

\section{References}

1. M. Johansson and A. Hunt, J. Coat. Technol. 67, (1995) 35.

2. (a) W. Shi and B. Ranby, J. Appl. Polym. Sci. 59 (1995) 1937. (b) W. Shi and B. Ranby, J. Appl. Polym. Sci. 59 (1995) 1945. (c) K. Maruyama, H. Kudo, T. Ikehara, N. Ito and T. Nishikubo, J. Polym. Sci. Polym. Chem. 43 (2005) 4642. (d) K. Maruyama, H. Kudo, T. Ikehara and T. Nishikubo, Macromolecules 40 (2007) 4895.

3. (a) L. Fogelström, P. Antoni, E. Malmström and A. Hult, Prog. Org. Coat. 55 (2006) 284. (b) H. Miao, L. Cheng, W. Shi, Prog. Org. Coat. 65 (2009) 71.

4. E. Žagar, M. Huskić and M. Žigon, Macromol. Chem. Phys. 208 (2007) 1379; references therein.

5. K. Aoki, and K. Ichimura, Chem. Lett. 38 (2009) 990. 